\title{
Molecular Targets and Emerging Therapeutic Options for Uterine Leiomyosarcoma
}

\author{
Heather Miller, ${ }^{1}$ Chiemeka Ike, ${ }^{2}$ Jennifer Parma, ${ }^{2}$ Ramya P. Masand, ${ }^{3}$ \\ Claire M. Mach, ${ }^{1,2}$ and Matthew L. Anderson ${ }^{1,3,4}$ \\ ${ }^{1}$ Department of Obstetrics \& Gynecology, Baylor College of Medicine, Houston, TX 77030, USA \\ ${ }^{2}$ College of Pharmacy, University of Houston, Houston, TX 77030, USA \\ ${ }^{3}$ Department of Pathology \& Immunology, Baylor College of Medicine, Houston, TX 77030, USA \\ ${ }^{4}$ Dan L Duncan Cancer Center, Baylor College of Medicine, Houston, TX 77030, USA \\ Correspondence should be addressed to Matthew L. Anderson; matthew@bcm.edu
}

Received 3 June 2016; Revised 5 August 2016; Accepted 18 August 2016

Academic Editor: Chandrajit Premanand Raut

Copyright (C) 2016 Heather Miller et al. This is an open access article distributed under the Creative Commons Attribution License, which permits unrestricted use, distribution, and reproduction in any medium, provided the original work is properly cited.

\begin{abstract}
Uterine leiomyosarcoma (uLMS) is an aggressive malignancy characterized by its early metastasis, high rates of recurrence, and poor prognosis. Multiple obstacles complicate the clinical management of uLMS. These include the fact that most uLMS are typically identified only after a woman has undergone hysterectomy or myomectomy, the limited efficacy of adjuvant therapy for early stage disease, and the poor response of metastatic disease to current treatments. Here, we discuss recent insights into the molecular basis of uLMS and discuss emerging options for its clinical management. Particular attention is given to the biologic basis of these strategies with the goal of understanding the rationale motivating their use.
\end{abstract}

\section{Introduction}

Uterine leiomyosarcoma (uLMS) is an aggressive cancer characterized by its poor prognosis and high rates of recurrence $[1,2]$. Although fewer than 3,000 women are diagnosed with this disease in the United States each year, uLMS is the most common sarcoma arising in the genital tract of reproductiveaged women $[3,4]$.

A number of long-standing challenges complicate clinical management of uLMS. Perhaps one of the most important of these challenges is the fact that uLMS are typically diagnosed only after a woman has undergone hysterectomy or myomectomy. This problem occurs for a number of reasons. The most important of these reasons is that strategies used to identify other types of uterine cancer, such as endometrial biopsy, are not useful for diagnosing this disease $[5,6]$. Currently, uLMS are diagnosed on the basis of the histologic identification of a high mitotic count (>10 mitotic figures/10 high power fields) and the presence of coagulative tumor necrosis and moderate to severe cytologic atypia [7]. Unfortunately, these features cannot be sufficiently evaluated by small volume core biopsies or needle aspirations to differentiate malignant from nonmalignant myometrial tissue. In addition, symptoms associated with uLMS, such as irregular vaginal bleeding or pelvic pain, are nonspecific and frequently caused by multiple, more common but benign etiologies [8]. A rapidly growing myometrial mass is often presumed to be pathognomonic, although existing data fail to support this belief [9].

Recognizing a clear area of clinical need, investigators have explored the utility of different imaging modalities to distinguish uLMS from benign leiomyomas preoperatively. Although a number of specific radiographic features, such as infiltrative margins, have been associated with uLMS visualized by magnetic resonance imaging (MRI), these features are not found frequently enough to allow routine use of MRI to prospectively distinguish benign from malignant myometrial masses [10]. Furthermore, there are no radiographic features capable of reliably distinguishing uLMS from benign myometrial masses by pelvic ultrasonography, computed tomography (CT), or positron emission tomography (PET).

A second key clinical challenge complicating management of uLMS is the need for effective adjuvant therapy 




FIGURE 1: Graphic summary of emerging and potential future targeted therapy for uLMS.

following hysterectomy. Most uLMS (68\%) are diagnosed as a solitary mass grossly confined to the uterus, which is defined as stage I disease according to the 2009 revised FIGO criteria [11]. Recurrence rates even for early stage disease are high, ranging from 53 to $71 \%[12,13]$. As a result, three-year survival for FIGO stage I uLMS is estimated to be only $52 \%$ [1]. Unfortunately, surgical staging is largely unable to identify women at risk of experiencing a recurrence of their disease. In the absence of grossly visible metastases, routine pelvic and paraaortic lymphadenectomy identifies microscopic metastases in only $2-3 \%$ of cases $[14,15]$. Routine oophorectomy similarly fails to provide prognostic insight. In fact, oophorectomy has been associated with worse overall survival, although data addressing this issue remains conflicted [16].

Given the high recurrence rates associated with early stage disease, adjuvant therapy is frequently administered to women who have recently undergone hysterectomy for uLMS. Multiple, early retrospective studies promoted the use of adjuvant radiotherapy to reduce the incidence of disease recurrence $[17,18]$. However, at least one recent prospective randomized control trial has failed to demonstrate any improvement in progression-free or overall survival for women with early stage uterine sarcomas including uLMS treated with radiotherapy $[1,19]$. As a result, use of adjuvant radiotherapy has largely been abandoned. Several large retrospective studies have suggested that adjuvant chemotherapy may also provide little benefit. The role of adriamycin as adjuvant therapy following surgical management of stage I or II disease has been studied with no difference in PFS or OS observed [20]. More recently, a phase III clinical trial compared the uses of doxorubicin, ifosfamide, and cisplatin as adjuvant therapy with and without radiotherapy for uterine sarcoma. Data from this study revealed a slight increase in 3-year disease-free survival in subjects who received both chemotherapy and radiotherapy [21]. Another phase II clinical trial examined the efficacy of adjuvant gemcitabine and docetaxel in patients with completely resected stage I and II disease. The outcome of this study demonstrated $57 \%$ progression-free survival (PFS) at 3 years, which was significantly greater than the 35\% PFS observed at 3 years among historical controls $[11,22]$. Despite the fact that each of these studies demonstrated modest improvements in PFS associated with the use of adjuvant chemotherapy, none of the regimens studied to date have yet to emerge as a consistent standard.

A final challenge is the treatment of advanced stage and/or recurrent uLMS. Similar to early stage disease, doxorubicin with or without ifosfamide has been historically used as frontline therapy for advanced or recurrent disease. However, response rates to doxorubicin-based strategies range from 15 to $30 \%$ [23]. A more recent phase II study has shown that the combination of gemcitabine and docetaxel may be more effective, with response rates reported to be as high as $36 \%[24,25]$. The use of gemcitabine and docetaxel as first line therapy has been prospectively compared to doxorubicin as part of a phase III randomized trial (GeDDIS) enrolling patients with metastatic soft tissue sarcomas. Although similar progression-free survival rates were observed in both treatment arms, subjects treated with doxorubicin experienced significantly less toxicity. Study investigators concluded that doxorubicin should remain first line treatment for patients with metastatic soft tissue sarcomas [26].

Given these challenges, there is an overwhelming need to develop more effective strategies for the diagnosis and treatment of uLMS. Solutions will be made more difficult by the fact that uLMS now appears to be a unique biologic entity which responds differently to treatments than leiomyosarcomas arising at extrauterine sites [27-30]. Here, we review recent advances in the clinical management of uterine leiomyosarcoma, emphasizing the biologic basis of emerging therapeutic options (Figure 1). 


\section{Novel Targeted Therapies}

2.1. Tyrosine Kinase Inhibitors. Uterine leiomyosarcoma is a highly vascular cancer that expresses significantly greater levels of vascular endothelial growth factor (VEGF) expression than many other tumors [31]. The overexpression of VEGF in uLMS has been shown to correlate with higher tumor grade, disease metastasis, and decreased survival [32,33]. Although multiple antiangiogenic agents are now available for clinical use, the response of uLMS to many of these agents, such as bevacizumab, has been disappointing [34, 35]. A recent phase III clinical trial compared treatment with gemcitabine plus docetaxel with or without bevacizumab as first line therapy for advanced stage or recurrent uLMS. The results of this study failed to demonstrate any improvement in either progression-free or overall survival for patients who received bevacizumab [35].

More recently, results of randomized clinical trials have shown that a novel multityrosine kinase inhibitor known as pazopanib may be effective in treating soft tissue sarcomas. Pazopanib differs from bevacizumab in that it targets and inhibits not only the inherent tyrosine kinase activity of the VEGF receptor, but also those of the PDGF receptor and stem cell factor receptor (c-kit) [36]. Overexpression of EGFR, PDGFR- $\alpha$, PDGFR- $\beta$, and PDGF-B has been reported to be a feature of uLMS [29]. Furthermore, levels of PDGF-B expression in uLMS specimens from the same study have been shown to correlate with outcome [29]. These observations suggest that the activation of PDGF-regulated pathways plays a particularly important role in promoting the growth and metastasis of uLMS.

Recently, the European Organization for the Research and Treatment of Cancer study concluded a phase III clinical trial entitled PALETTE, which compared pazopanib to placebo as a treatment for soft tissue sarcomas. Patients diagnosed with both uterine and nonuterine leiomyosarcomas were enrolled. In this multicenter, double blind study, patients with progressive disease were randomized to receive either pazopanib $800 \mathrm{mg}$ once daily or placebo. Investigators reported a 3-month increase in progression-free survival for patients who received pazopanib [37].

Regorafenib is a multikinase inhibitor with documented efficacy for the treatment of gastrointestinal stromal tumors. Regorafenib has also recently been studied as a treatment for LMS [38]. A phase II double-blinded, randomized control trial compared regorafenib to placebo in patients with metastatic soft tissue sarcomas previously treated with anthracycline chemotherapy. In the subgroup of LMS patients enrolled in this study, PFS was found to be 4.0 months in patients receiving regorafenib compared to 1.9 months in the placebo arm. The most common adverse events noted were hypertension, skin toxicity, asthenia, and diarrhea $[39,40]$.

Olaratumab is a human antiplatelet derived growth factor receptor alpha monoclonal antibody that has been shown to have antitumor effects in human sarcoma xenografts [41]. Olaratumab specifically binds PDGFR $\alpha$ and blocks activation of this receptor by PDGF-AA, PDGF-BB, and PDGF-CC. A phase II randomized control trial compared the use of doxorubicin with and without olaratumab in patients with soft tissue sarcoma who had not previously been treated with an anthracycline. Subjects treated with doxorubicin and olaratumab had PFS of 6.6 months compared to 4.1 months with doxorubicin alone. Overall survival was better for subjects treated with the combination of doxorubicin and olaratumab (26.5 months compared to 14.7 months in those treated with doxorubicin alone). Neutropenia, mucositis, nausea, vomiting, and diarrhea each occurred more commonly in those subjects treated with olaratumab [42]. A phase III clinical trial is currently ongoing.

2.2. Antihormonal Agents. Reflecting their origin in the female reproductive tract, gynecologic sarcomas often express receptors for estrogen and progesterone. When examined immunohistochemically, 25-60\% of uLMS have been found to express estrogen receptor (ER), while $35-60 \%$ of cases express progesterone receptor (PR) [43, 44].

Multiple studies have shown that estrogen and progesterone receptor expression in uLMS correlates with improved prognosis [43, 45, 46]. However, at least one group has suggested that activation of PR may serve to promote the growth of uterine smooth muscle [47]. Transgenic expression of Simian Virus 40 large $\mathrm{T}$ antigen driven by the promoter for oviduct-specific glycoprotein has been recently shown to result in the development of leiomyosarcoma [48, 49]. Of note, these transgenic animals develop LMS only under the influence of estrogen. These findings suggest that circulating levels of ligand-specific ER activation play an important role in tumor development.

Options currently available to inhibit activity at both the estrogen (ER) and progesterone (PR) receptors include both direct receptor antagonists as well as aromatase inhibitors. A number of studies have also examined the efficacy of direct ER antagonists such as tamoxifen. However, response to tamoxifen appears quite limited [44]. Aromatase inhibitors are orally available medications that decrease circulating levels of estrogen by targeting the peripheral conversion of testosterone and androstenedione to estrogen in adipose tissue. Retrospective studies have shown objective response rates and improvement in progression-free survival in patients with uLMS treated with aromatase inhibitors such as anastrozole or letrozole $[50,51]$. A phase II clinical trial was conducted that examined the effect of letrozole $2.5 \mathrm{mg}$ daily in 27 patients with unresectable uLMS, most of whom had already received cytotoxic treatment. All patients had confirmed ER/PR expression as evidenced by immunohistochemical staining. Stable disease was observed in 54\% of patients receiving letrozole, with no evidence of disease progression at 12 weeks for $46 \%$ of patients [52].

Given the central role of both ER and PR in regulating the growth and remodeling of uterine smooth muscle, future work aimed at understanding the potential role of targeting steroid hormone receptors and/or their coactivators or corepressors as therapeutic options in uLMS are clearly warranted. Better understanding of the role of ER and PR in this disease may also help to decipher the circumstances under which oophorectomy may benefit the outcome. 
2.3. Next Generation Alkylating Agents. As mentioned above, doxorubicin and other alkylating agents have been one of the primary approaches used to treat both metastatic and recurrent uLMS $[23,53]$. Over the past 5 years, use of these agents has declined, with clinicians more frequently favoring the use of other regimens. However, recent success with orally available alkylating agents, such as temozolomide, has precipitated renewed interest in their use in the treatment of soft tissue sarcomas $[54,55]$. A small retrospective study of 12 women with unresectable disease compared the efficacy of two dosing regimens for temozolomide in uLMS. Response rates appeared higher in patients treated with a bolus regimen (14\%) rather than with a continuous dosing regimen (8\%) [56]. A recent report has found that O6-methylguanineDNA methyltransferase (MGMT) gene promoter hypermethylation is associated with response of glioblastoma to temozolomide therapy and may be a key factor determining a response to this agent. In a number of cancers, promoter hypermethylation has been shown to result in decreased expression of MGMT and gene as well as diminished DNA repair activity, rendering cells more susceptible to alkylating agents [57]. Hypermethylation of the MGMT gene promoter has also been observed in uterine sarcomas and thus could serve as a potential marker to identify patients who would benefit from temozolomide use $[58,59]$.

Trabectedin is another novel alkylating agent that has been recently approved in the United States for use in treating unresectable uLMS that have progressed despite receiving a standard cytotoxic chemotherapy regimen, which included an anthracycline. Trabectedin is a marine-derived alkaloid that interacts with the minor groove of DNA, leading to apoptosis [60]. Studies have examined the use of trabectedin alone and in combination with other chemotherapeutic agents. A phase II study examining the use of trabectedin as a single agent in chemotherapy naïve patients with advanced uLMS reported a $10 \%$ partial response rate [61]. However, trabectedin must be continued in absence of progression of disease, as discontinuation of the drug is associated with decreased progression-free survival. Another phase II study examined the efficacy of first line therapy with the combination of trabectedin and doxorubicin and reported a partial response rate of $60 \%$ [62]. The role of MGMT promoter hypermethylation in determining clinical responses to trabectedin is not currently known.

2.4. mTOR Inhibition. mTOR is a serine/threonine kinase involved in cell growth, proliferation, survival, and metabolism [63]. mTOR activation has been shown to contribute to uLMS cell growth and cell cycle progression. Specifically, loss of PTEN (phosphatase and tensin homolog) results in AKT-mTOR activation. In transgenic mice, inactivation or loss of PTEN leads to the development of uLMS $[64,65]$. Rapamycin blocks the mTOR pathway and has been shown to have antitumor effects in preclinical models and has been shown to inhibit growth and cell cycle progression in uLMS cell lines $[66,67]$. In PTEN knockout mice, everolimus, a rapamycin derivative, was shown to decelerate tumor growth [64]. mTOR inhibitors have been used clinically for treatment of soft tissue sarcomas [68]. Additional work is needed to determine the efficacy of mTOR inhibitors in uLMS specifically.

\section{Novel Targets with Potential for Future Utility}

3.1. Cell Cycle Inhibitors. In contrast to tumors, such as gastrointestinal stromal sarcomas (GIST), leiomyosarcoma regardless of its anatomic origin is considered genetically heterogeneous. This means that dominant driver mutations for uLMS have not been identified. A number of recent studies have examined patterns of gene expression in LMS of both uterine and nonuterine origin. It is clear from this work that the robust overexpression of gene products regulating the G2-M phase of the cell cycle is single predominant feature of these tumors $[69,70]$. In one recent study, 26 of the 50 gene products most overexpressed when specimens of uLMS were compared to either healthy myometrium or benign uterine leiomyomas which played a role in regulation of the G2$\mathrm{M}$ checkpoint [70]. Aurora kinase-A (AurkA) is a serinethreonine kinase involved in centrosome function and spindle assembly and has been shown to be highly overexpressed in uLMS. Use of MLN82237, an oral Aurk-A inhibitor, has been found to inhibit growth and metastasis of uLMS both in vitro and in vivo, using xenograft models [69]. Additional therapeutic benefit was observed when MLN82237 was combined with mTOR inhibition, consistent with AurkA's ability to cross-regulate mTOR pathway activity $[67,71]$. A small, phase II clinical trial has examined the efficacy of MLN8237 (alisertib) monotherapy in 21 women with recurrent or persistent uLMS. All subjects in this study had received 1 to 2 prior cytotoxic regimens. Although no objective responses were reported, stable disease was observed in $38 \%$ of patients with mean progression-free and overall survival of 1.7 and 14.5 months, respectively [72]. Although the investigators for this trial concluded that alisertib did not demonstrate meaningful clinical activity against uLMS, it is possible that better efficacy will be observed when used in combination with mTOR inhibitors or even other types of cytotoxic or targeted agents.

3.2. Epigenetic Modifiers. Histone deacetylases (HDAC) are enzymes, which regulate patterns of gene expression modulating cell growth and apoptosis by removing acetyl moieties from histones [73, 74]. HDAC expression has been shown to be consistently elevated in endometrial stromal sarcomas [75]. Data has also shown that HDAC inhibitors can enhance chemotherapy-induced apoptosis and reduce sarcoma tumor volume in preclinical models [76]. Vorinostat is an oral HDAC inhibitor that has already been FDA approved for the treatment of cutaneous T cell lymphoma [77]. Studies have shown that vorinostat suppresses tumor growth in uterine sarcoma cells [76]. Given this preclinical data, HDAC inhibitors are being studied for targeted therapy in uterine sarcomas. A phase I trial evaluated the use of a HDAC inhibitor, abexinostat, in combination with doxorubicin for patients with metastatic sarcoma and showed manageable toxicities [78]. Further studies are needed to evaluate the 
efficacy of HDAC inhibitors in combination with cytotoxic agents and in uLMS specifically.

\section{Conclusion}

Management options for uLMS have evolved rapidly over the past several years with the advent of specific targeted therapies. These options have given patients new hope for improving their outcome. Given that uLMS likely represents a unique subset, distinct from extrauterine leiomyosarcomas, future therapeutic advances for this aggressive disease will need to consider its unique biologic basis.

\section{Competing Interests}

The authors have no conflict of interests to declare.

\section{References}

[1] N. S. Reed, C. Mangioni, H. Malmström et al., "Phase III randomised study to evaluate the role of adjuvant pelvic radiotherapy in the treatment of uterine sarcomas stages I and II: an European Organisation for Research and Treatment of Cancer Gynaecological Cancer Group Study (protocol 55874)," European Journal of Cancer, vol. 44, no. 6, pp. 808-818, 2008.

[2] F. J. Major, J. A. Blessing, S. G. Silverberg et al., "Prognostic factors in early-stage uterine sarcoma: A Gynecologic Oncology Group study," Cancer, vol. 71, no. 4, pp. 1702-1709, 1993.

[3] R. L. Siegel, K. D. Miller, and A. Jemal, "Cancer statistics, 2015," CA-A Cancer Journal for Clinicians, vol. 65, no. 1, pp. 5-29, 2015.

[4] S. M. Ueda, D. S. Kapp, M. K. Cheung et al., "Trends in demographic and clinical characteristics in women diagnosed with corpus cancer and their potential impact on the increasing number of deaths," American Journal of Obstetrics \& Gynecology, vol. 198, no. 2, pp. 218.e1-218.e6, 2008.

[5] N. Bansal, T. J. Herzog, W. Burke, C. J. Cohen, and J. D. Wright, "The utility of preoperative endometrial sampling for the detection of uterine sarcomas," Gynecologic Oncology, vol. 110, no. 1, pp. 43-48, 2008.

[6] E. M. Hinchcliff, K. M. Esselen, J. C. Watkins et al., “The role of endometrial biopsy in the preoperative detection of uterine leiomyosarcoma," Journal of Minimally Invasive Gynecology, vol. 23, no. 4, pp. 567-572, 2016.

[7] S. Chiang and E. Oliva, "Recent developments in uterine mesenchymal neoplasms," Histopathology, vol. 62, no. 1, pp. 124137, 2013.

[8] A. A. Gockley, J. A. Rauh-Hain, and M. G. Del Carmen, "Uterine leiomyosarcoma a review article," International Journal of Gynecological Cancer, vol. 24, no. 9, pp. 1538-1542, 2014.

[9] W. H. Parker, Y. S. Fu, and J. S. Berek, "Uterine sarcoma in patients operated on for presumed leiomyoma and rapidly growing leiomyoma," Obstetrics and Gynecology, vol. 83, no. 3, pp. 414-418, 1994.

[10] S. H. Tirumani, V. Ojili, A. K. P. Shanbhogue, N. Fasih, J. G. Ryan, and C. Reinhold, "Current concepts in the imaging of uterine sarcoma," Abdominal Imaging, vol.38, no. 2, pp. 397-411, 2013.

[11] M. L. Hensley, O. Zivanovic, M. M. Leitao et al., "Stagespecific outcomes of patients with Uterine leiomyosarcoma: a comparison of the international federation of gynecology and obstetrics and american joint committee on cancer staging systems," Journal of Clinical Oncology, vol. 27, no. 12, pp. 20662072, 2009.

[12] O. Zivanovic, L. M. Jacks, A. Iasonos et al., "A nomogram to predict postresection 5-year overall survival for patients with uterine leiomyosarcoma," Cancer, vol. 118, no. 3, pp. 660-669, 2012.

[13] A. Iasonos, E. Z. Keung, O. Zivanovic et al., "External validation of a prognostic nomogram for overall survival in women with uterine leiomyosarcoma," Cancer, vol. 119, no. 10, pp. 1816-1822, 2013.

[14] M. M. Leitao, Y. Sonoda, M. F. Brennan, R. R. Barakat, and D. S. Chi, "Incidence of lymph node and ovarian metastases in leiomyosarcoma of the uterus," Gynecologic Oncology, vol. 91, no. 1, pp. 209-212, 2003.

[15] K. Y. Tse, R. Crawford, and H. Y. S. Ngan, "Staging of uterine sarcomas," Best Practice \& Research Clinical Obstetrics \& Gynaecology, vol. 25, no. 6, pp. 733-749, 2011.

[16] D. S. Kapp, J. Y. Shin, and J. K. Chan, "Prognostic factors and survival in 1396 patients with uterine leiomyosarcomas: emphasis on impact of lymphadenectomy and oophorectomy," Cancer, vol. 112, no. 4, pp. 820-830, 2008.

[17] P. Wong, K. Han, J. Sykes et al., "Postoperative radiotherapy improves local control and survival in patients with uterine leiomyosarcoma," Radiation Oncology, vol. 8, no. 1, article 128, 2013.

[18] S. Sampath, T. E. Schultheiss, J. K. Ryu, and J. Y. C. Wong, "The role of adjuvant radiation in uterine sarcomas," International Journal of Radiation Oncology ${ }^{*}$ Biology ${ }^{*}$ Physics, vol. 76, no. 3, pp. 728-734, 2010.

[19] P. Pautier, A. Floquet, L. Gladieff et al., "A randomized clinical trial of adjuvant chemotherapy with doxorubicin, ifosfamide, and cisplatin in localized uterine sarcomas. Results on 81 randomized patients," European Journal of Cancer, vol. 47, supplement 2, p. 15, 2011.

[20] G. A. Omura, J. A. Blessing, F. Major et al., "A randomized clinical trial of adjuvant adriamycin in uterine sarcomas: a Gynecologic Oncology Group Study," Journal of Clinical Oncology, vol. 3, no. 9, pp. 1240-1245, 1985.

[21] P. Pautier, A. Floquet, L. Gladieff et al., "A randomized clinical trial of adjuvant chemotherapy with doxorubicin, ifosfamide, and cisplatin followed by radiotherapy versus radiotherapy alone in patients with localized uterine sarcomas (SARCGYN study). A study of the French sarcoma group," Annals of Oncology, vol. 24, no. 4, Article ID mds545, pp. 1099-1104, 2013.

[22] M. L. Hensley, J. K. Wathen, R. G. Maki et al., "Adjuvant therapy for high-grade, uterus-limited leiomyosarcoma: results of a phase 2 trial (SARC 005)," Cancer, vol. 119, no. 8, pp. 15551561, 2013.

[23] G. Sutton, J. A. Blessing, and J. H. Malfetano, "Ifosfamide and doxorubicin in the treatment of advanced leiomyosarcomas of the uterus: A Gynecologic Oncology Group study," Gynecologic Oncology, vol. 62, no. 2, pp. 226-229, 1996.

[24] M. L. Hensley, J. A. Blessing, R. Mannel, and P. G. Rose, "Fixeddose rate gemcitabine plus docetaxel as first-line therapy for metastatic uterine leiomyosarcoma: a Gynecologic Oncology Group phase II trial," Gynecologic Oncology, vol. 109, no. 3, pp. 329-334, 2008.

[25] R. G. Maki, J. K. Wathen, S. R. Patel et al., "Randomized phase II study of gemcitabine and docetaxel compared with gemcitabine 
alone in patients with metastatic soft tissue sarcomas," Journal of Clinical Oncology, vol. 25, no. 19, pp. 2755-2763, 2007.

[26] B. Seddon, J. Whelan, S. Strauss et al., "A prospective randomized controlled phase III trial of gemcitabine and docetaxel compared wiht doxorubicin as first-line treatment in previously untreated advanced unresectable or metastatic soft tissue sarcomas," Journal of Clinical Oncology, vol. 33, supplement, abstract 10500, 2015.

[27] W. Lamm, C. Natter, S. Schur et al., "Distinctive outcome in patients with non-uterine and uterine leiomyosarcoma," BMC Cancer, vol. 14, no. 1, article 981, 2014.

[28] D. J. Worhunsky, M. Gupta, S. Gholami et al., "Leiomyosarcoma: one disease or distinct biologic entities based on site of origin?" Journal of Surgical Oncology, vol. 111, no. 7, pp. 808-812, 2015.

[29] K. Lusby, K. B. Savannah, E. G. Demicco et al., "Uterine leiomyosarcoma management, outcome, and associated molecular biomarkers: a single institution's experience," Annals of Surgical Oncology, vol. 20, no. 7, pp. 2364-2372, 2013.

[30] X. Guo, V. Y. Jo, A. M. Mills et al., "Clinically relevant molecular subtypes in leiomyosarcoma," Clinical Cancer Research, vol. 21, no. 15, pp. 3501-3511, 2015.

[31] S. S. Yoon, N. H. Segal, A. B. Olshen, M. F. Brennan, and S. Singer, "Circulating angiogenic factor levels correlate with extent of disease and risk of recurrence in patients with soft tissue sarcoma," Annals of Oncology, vol. 15, no. 8, pp. 1261-1266, 2004.

[32] D. S. McMeekin, M. W. Sill, K. M. Darcy et al., "A phase II trial of thalidomide in patients with refractory leiomyosarcoma of the uterus and correlation with biomarkers of angiogenesis: A Gynecologic Oncology Group Study," Gynecologic Oncology, vol. 106, no. 3, pp. 596-603, 2007.

[33] U. Graeven, N. Andre, E. Achilles, C. Zornig, and W. Schmiegel, "Serum levels of vascular endothelial growth factor and basic fibroblast growth factor in patients with soft-tissue sarcoma," Journal of Cancer Research and Clinical Oncology, vol. 125, no. 10, pp. 577-581, 1999.

[34] S. Pacey, M. J. Ratain, K. T. Flaherty et al., "Efficacy and safety of sorafenib in a subset of patients with advanced soft tissue sarcoma from a phase II randomized discontinuation trial," Investigational New Drugs, vol. 29, no. 3, pp. 481-488, 2011.

[35] M. L. Hensley, A. Miller, D. M. O’Malley et al., "Randomized phase III trial of gemcitabine plus docetaxel plus bevacizumab or placebo as first-line treatment for metastatic uterine leiomyosarcoma: An NRG Oncology/ Gynecologic Oncology Group Study," Journal of Clinical Oncology, vol. 33, no. 10, pp. 1180-1185, 2015.

[36] J.-M. Schlaeppi and J. M. Wood, "Targeting vascular endothelial growth factor (VEGF) for anti-tumor therapy, by antiVEGF neutralizing monoclonal antibodies or by VEGF receptor tyrosine-kinase inhibitors," Cancer and Metastasis Reviews, vol. 18, no. 4, pp. 473-481, 1999.

[37] W. T. A. Van Der Graaf, J.-Y. Blay, S. P. Chawla et al., "Pazopanib for metastatic soft-tissue sarcoma (PALETTE): a randomised, double-blind, placebo-controlled phase 3 trial," The Lancet, vol. 379, no. 9829, pp. 1879-1886, 2012.

[38] K. A. Lyseng-Williamson, "Regorafenib: a guide to its use in advanced gastrointestinal stromal tumor (GIST) after failure of imatinib and sunitinib," BioDrugs, vol. 27, no. 5, pp. 525-531, 2013.

[39] O. Mir, T. Brodowicz, J. Wallet et al., "Activity of regorafenib (RE) in leiomyosarcomas (LMS) and other types of soft-tissue sarcomas (OTS): results of a double-blind, randomized placebo (PL) controlled phase II trial," Journal of Clinical Oncology, vol. 33, no. 15, supplement 1, 2015.

[40] T. Brodowicz, B. Liegl-Atzwager, E. Tresch et al., "Study protocol of REGOSARC trial: activity and safety of regorafenib in advanced soft tissue sarcoma: a multinational, randomized, placebo-controlled, phase II trial," BMC Cancer, vol. 15, article 127, 2015.

[41] N. Loizos, Y. Xu, J. Huber et al., “Targeting the plateletderived growth factor receptor $\alpha$ with a neutralizing human monoclonal antibody inhibits the growth of tumor xenografts: implications as a potential therapeutic target," Molecular Cancer Therapeutics, vol. 4, no. 3, pp. 369-379, 2005.

[42] W. D. Tap, R. L. Jones, B. A. Van Tine et al., "Olaratumab and doxorubicin versus doxorubicin alone for treatment of softtissue sarcoma: an open-label phase $1 \mathrm{~b}$ and randomised phase 2 trial," The Lancet, vol. 388, no. 10043, pp. 488-497, 2016.

[43] K. Bodner, B. Bodner-Adler, O. Kimberger, K. Czerwenka, S. Leodolter, and K. Mayerhofer, "Estrogen and progesterone receptor expression in patients with uterine leiomyosarcoma and correlation with different clinicopathological parameters," Anticancer Research, vol. 23, no. 1, pp. 729-732, 2003.

[44] Y. J. Ioffe, A. J. Li, C. S. Walsh et al., "Hormone receptor expression in uterine sarcomas: prognostic and therapeutic roles," Gynecologic Oncology, vol. 115, no. 3, pp. 466-471, 2009.

[45] S. E. Akhan, E. Yavuz, A. Tecer et al., "The expression of Ki-67, p53, estrogen and progesterone receptors affecting survival in uterine leiomyosarcomas. A Clinicopathologic Study," Gynecologic Oncology, vol. 99, no. 1, pp. 36-42, 2005.

[46] M. M. Leitao Jr., M. L. Hensley, R. R. Barakat et al., "Immunohistochemical expression of estrogen and progesterone receptors and outcomes in patients with newly diagnosed uterine leiomyosarcoma," Gynecologic Oncology, vol. 124, no. 3, pp. 558$562,2012$.

[47] J. J. Kim, T. Kurita, and S. E. Bulun, "Progesterone action in endometrial cancer, endometriosis, uterine fibroids, and breast cancer," Endocrine Reviews, vol. 34, no. 1, pp. 130-162, 2013.

[48] I. Miyoshi, K. Takahashi, Y. Kon et al., "Mouse transgenic for murine oviduct-specific glycoprotein promoter-driven simian virus 40 large T-antigen: tumor formation and its hormonal regulation," Molecular Reproduction and Development, vol. 63, no. 2, pp. 168-176, 2002.

[49] A. M. Friel, W. B. Growdon, C. K. McCann et al., "Mouse models of uterine corpus tumors: clinical significance and utility," Frontiers in Bioscience, vol. 2, pp. 882-905, 2010.

[50] R. O'Cearbhaill, Q. Zhou, A. Iasonos et al., "Treatment of advanced uterine leiomyosarcoma with aromatase inhibitors," Gynecologic Oncology, vol. 116, no. 3, pp. 424-429, 2010.

[51] E. Thanopoulou, K. Thway, K. Khabra, and I. Judson, "Treatment of hormone positive uterine leiomyosarcoma with aromatase inhibitors," Clinical Sarcoma Research, vol. 4, no. 1, article 5, 2014.

[52] S. George, Y. Feng, J. Manola et al., "Phase 2 trial of aromatase inhibition with letrozole in patients with uterine leiomyosarcomas expressing estrogen and/or progesterone receptors," Cancer, vol. 120, no. 5, pp. 738-743, 2014.

[53] S. Sleijfer, C. Seynaeve, and J. Verweij, "Using single-agent therapy in adult patients with advanced soft tissue sarcoma can still be considered standard care," Oncologist, vol. 10, no. 10, pp. 833-841, 2005. 
[54] S. M. Talbot, M. L. Keohan, M. Hesdorffer et al., "A phase II trial of temozolomide in patients with unresectable or metastatic soft tissue sarcoma," Cancer, vol. 98, no. 9, pp. 1942-1946, 2003.

[55] P. J. Woll, I. Judson, S. M. Lee et al., "Temozolomide in adult patients with advanced soft tissue sarcoma: a phase II study of the EORTC soft tissue and bone sarcoma group," European Journal of Cancer, vol. 35, no. 3, pp. 410-412, 1999.

[56] S. Anderson and C. Aghajanian, "Temozolomide in uterine leiomyosarcomas," Gynecologic Oncology, vol. 98, no. 1, pp. 99103, 2005.

[57] M. E. Hegi, A.-C. Diserens, T. Gorlia et al., "MGMT gene silencing and benefit from temozolomide in glioblastoma," The New England Journal of Medicine, vol. 352, no. 10, pp. 997-1003, 2005.

[58] M. Bujko, M. Kowalewska, A. Danska-Bidzinska, E. BakulaZalewska, J. A. Siedecki, and M. Bidzinski, "The promoter methylation and expression of the O6-methylguanine-DNA methyltransferase gene in uterine sarcoma and carcinosarcoma," Oncology Letters, vol. 4, no. 3, pp. 551-555, 2012.

[59] J. S. Ferriss, K. A. Atkins, J. A. Lachance, S. C. Modesitt, and A. A. Jazaeri, "Temozolomide in advanced and recurrent uterine leiomyosarcoma and correlation with O6-methylguanine DNA methyltransferase expression a case series," International Journal of Gynecological Cancer, vol. 20, no. 1, pp. 120-125, 2010.

[60] M. D'Incalci and C. M. Galmarini, "A review of trabectedin (ET-743): a unique mechanism of action," Molecular Cancer Therapeutics, vol. 9, no. 8, pp. 2157-2163, 2010.

[61] B. J. Monk, J. A. Blessing, D. G. Street, C. Y. Muller, J. J. Burke, and M. L. Hensley, "A phase II evaluation of trabectedin in the treatment of advanced, persistent, or recurrent uterine leiomyosarcoma: A Gynecologic Oncology Group Study," Gynecologic Oncology, vol. 124, no. 1, pp. 48-52, 2012.

[62] P. Pautier, A. Floquet, C. Chevreau et al., "Trabectedin in combination with doxorubicin for first-line treatment of advanced uterine or soft-tissue leiomyosarcoma (LMS-02): a non-randomised, multicentre, phase 2 trial," The Lancet Oncology, vol. 16, no. 4, pp. 457-464, 2015.

[63] D. D. Sarbassov, D. A. Guertin, S. M. Ali, and D. M. Sabatini, "Phosphorylation and regulation of Akt/PKB by the rictormTOR complex," Science, vol. 307, no. 5712, pp. 1098-1101, 2005.

[64] E. Hernando, E. Charytonowicz, M. E. Dudas et al., "The AKTmTOR pathway plays a critical role in the development of leiomyosarcomas," Nature Medicine, vol. 13, no. 6, pp. 748-753, 2007.

[65] T. F. Wong, T. Takeda, B. Li et al., "Curcumin disrupts uterine leiomyosarcoma cells through AKT-mTOR pathway inhibition," Gynecologic Oncology, vol. 122, no. 1, pp. 141-148, 2011.

[66] X. Wan and L. J. Helman, "The biology behind mTOR inhibition in sarcoma," Oncologist, vol. 12, no. 8, pp. 1007-1018, 2007.

[67] K. J. Brewer Savannah, E. G. Demicco, K. Lusby et al., "Dual targeting of mTOR and Aurora-A kinase for the treatment of uterine leiomyosarcoma," Clinical Cancer Research, vol. 18, no. 17, pp. 4633-4645, 2012.

[68] S. Vemulapalli, A. Mita, Y. Alvarado, K. Sankhala, and M. Mita, "The emerging role of mammalian target of rapamycin inhibitors in the treatment of sarcomas," Targeted Oncology, vol. 6, no. 1, pp. 29-39, 2011.

[69] W. Shan, P. Y. Akinfenwa, K. B. Savannah et al., "A smallmolecule inhibitor targeting the mitotic spindle checkpoint impairs the growth of uterine leiomyosarcoma," Clinical Cancer Research, vol. 18, no. 12, pp. 3352-3365, 2012.
[70] J. N. Barlin, Q. C. Zhou, M. M. Leitao et al., "Molecular subtypes of uterine leiomyosarcoma and correlation with clinical outcome," Neoplasia, vol. 17, no. 2, pp. 183-189, 2015.

[71] X. Wang, Y.-X. Zhou, W. Qiao et al., "Overexpression of aurora kinase A in mouse mammary epithelium induces genetic instability preceding mammary tumor formation," Oncogene, vol. 25, no. 54, pp. 7148-7158, 2006.

[72] D. M. Hyman, M. Sill, J. K. Cho et al., "A phase II study of alisertib (MLN8237) in recurrent or persistent uterine leiomyosarcoma: an NRG Oncology/Gynecologic Oncology Group study (GOG-0231D)," Journal of Clinical Oncology, vol. 33, supplement, abstract e16512, 2015, Proceedings of the 2015 ASCO Annual Meeting.

[73] M. Dokmanovic, C. Clarke, and P. A. Marks, "Histone deacetylase inhibitors: overview and perspectives," Molecular Cancer Research, vol. 5, no. 10, pp. 981-989, 2007.

[74] W. S. Xu, R. B. Parmigiani, and P. A. Marks, "Histone deacetylase inhibitors: molecular mechanisms of action," Oncogene, vol. 26, no. 37, pp. 5541-5552, 2007.

[75] A. Hrzenjak, F. Moinfar, M.-L. Kremser et al., "Valproate inhibition of histone deacetylase 2 affects differentiation and decreases proliferation of endometrial stromal sarcoma cells," Molecular Cancer Therapeutics, vol. 5, no. 9, pp. 2203-2210, 2006.

[76] A. Hrzenjak, F. Moinfar, M.-L. Kremser et al., "Histone deacetylase inhibitor vorinostat suppresses the growth of uterine sarcomas in vitro and in vivo," Molecular Cancer, vol. 9, article 49, 2010.

[77] M. Duvic and J. Vu, "Vorinostat: a new oral histone deacetylase inhibitor approved for cutaneous T-cell lymphoma," Expert Opinion on Investigational Drugs, vol. 16, no. 7, pp. 1111-1120, 2007.

[78] E. Choy, Y. Flamand, S. Balasubramanian et al., "Phase 1 study of oral abexinostat, a histone deacetylase inhibitor, in combination with doxorubicin in patients with metastatic sarcoma," Cancer, vol. 121, no. 8, pp. 1223-1230, 2015. 


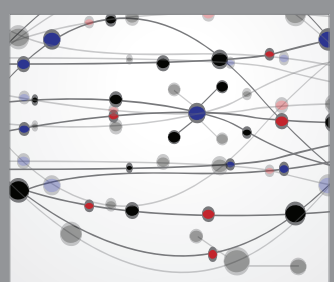

The Scientific World Journal
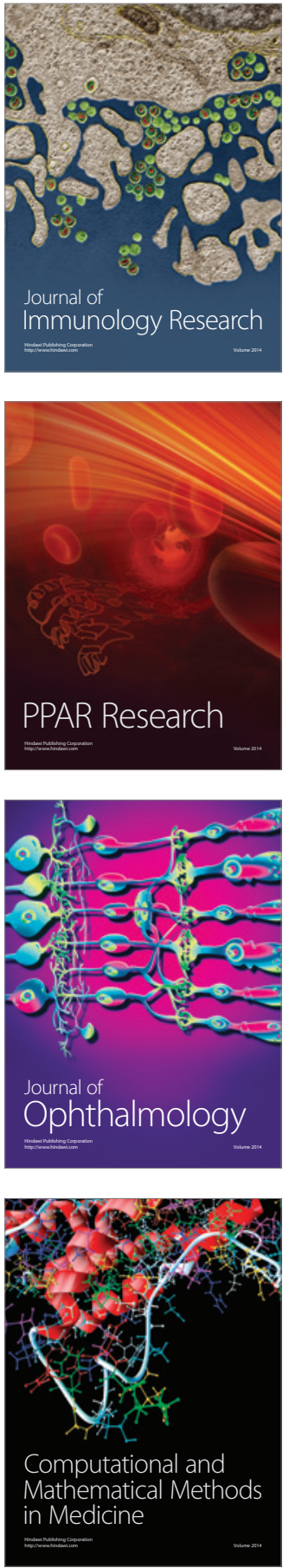

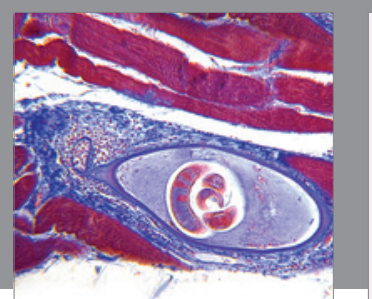

Gastroenterology Research and Practice

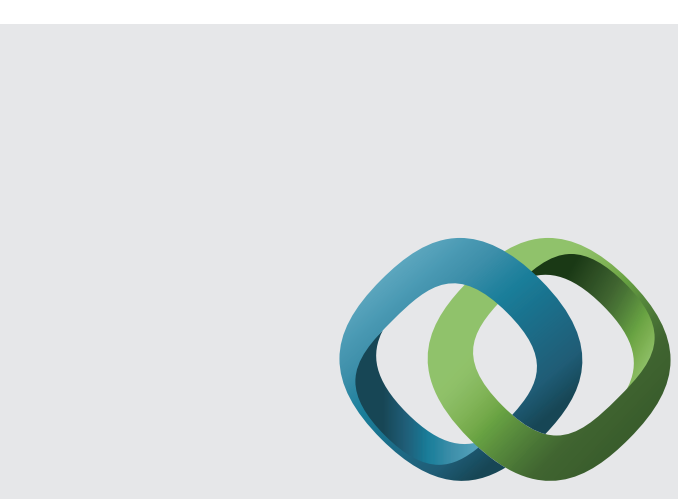

\section{Hindawi}

Submit your manuscripts at

http://www.hindawi.com
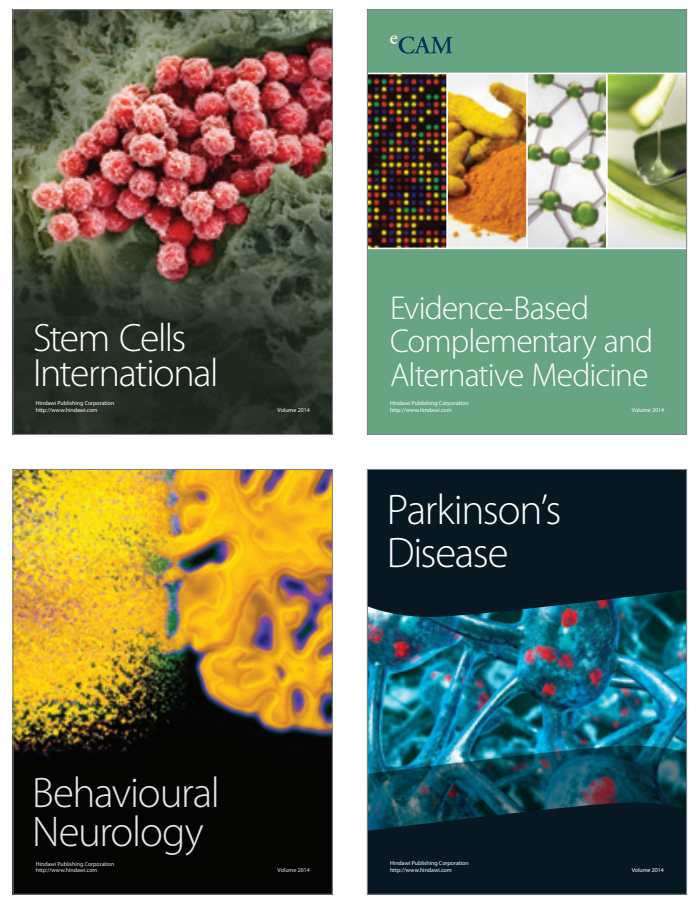
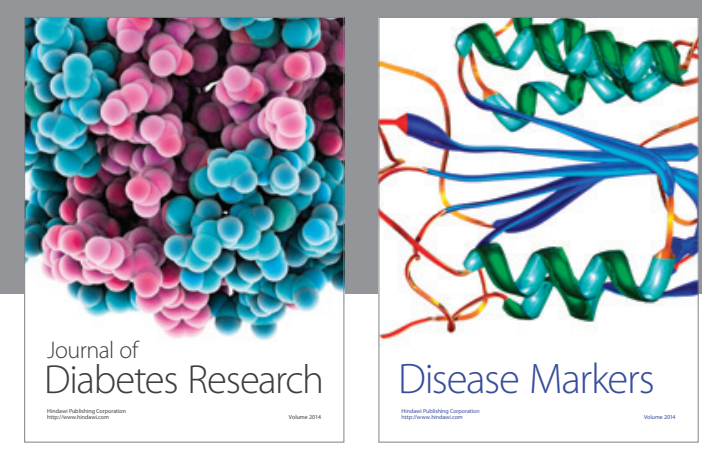

Disease Markers
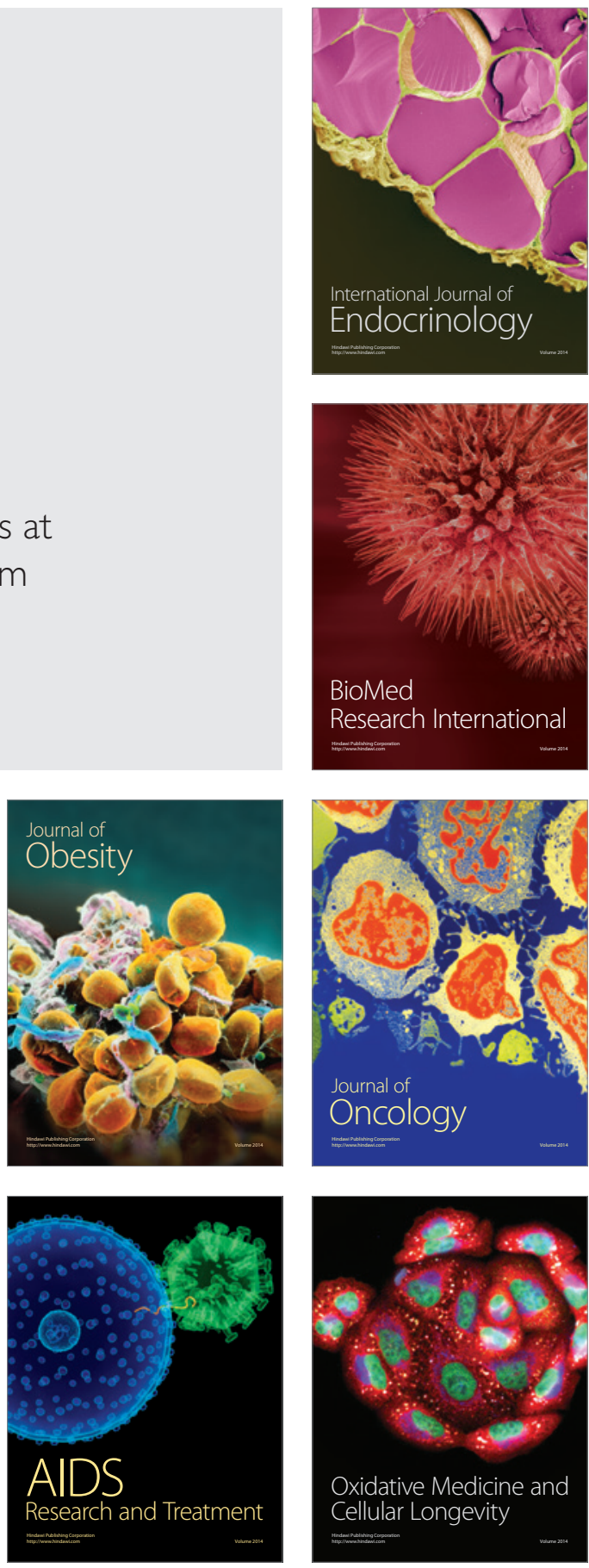